\title{
State-of-charge Estimation of Lithium-ion Battery Based Online Parameter Identification
}

\author{
Juqiang Feng ${ }^{l, *}$, Long $W u^{l}$, Kaifeng Huang ${ }^{l}$, Xing Zhang ${ }^{l}$, and Jun Lu ${ }^{2}$ \\ ${ }^{1}$ Engineering Technology Research Center of Rail Transit Power and Control, Huainan Normal University, 232038, Huainan, China \\ ${ }^{2}$ Manager of Technical Department, Huainan Research Institute of Mine electronic Technology, 232038, Huainan, China
}

\begin{abstract}
Accurately estimating the state of charge (SOC) of lithium-ion is very important to improving the dynamic performance and energy utilization efficiency. In order to reduce the influence of model parameters and system coloured noise on SOC estimation accuracy, this paper proposes the SOC estimation based on online identification. Based on the mixed simplified electrochemical model, the forgetting factor recursive least squares (FFRLS) method was used to identify the parameters online, and the SOC estimation was carried out in combination with Unscented Kalman Filter (UKF). Finally, the accuracy and feasibility of the method are verified by Federal Urban Driving Schedule (FUDS), the online identification and SOC estimation are carried out. The experimental results show that the SOC estimation of online parameter identification is more accurate, the system stability is faster and the error is smaller.
\end{abstract}

\section{Introduction}

Lithium-ion batteries are widely used in many applications, such as power grids, new energy vehicles, energy storage systems and various electronic devices, and play a vital role [1-2]. The usage of lithium-ion cells has fuelled growth in many applications because of high energy density, high power ratio, long cycling life (more than 500 times) and low self-discharge (less than $5 \%$ per month) [3]. Due to the use of new materials, high energy density can effectively reduce the volume and weight, while improving the circularity and speed capacity, which is highly attractive for hybrid electric vehicles.

In order to improve the energy utilization rate of lithium -ion batteries and realize safe management, accurate estimation of the charged state of lithium-ion batteries is very important. The charging and discharging process of lithium-ion batteries is a complex electrochemical system with strong nonlinear characteristics. Many researchers have used equivalent circuit model to characterize it, and the validity and practicability of the method have been proved [4]. However, the identification method of battery model parameters directly affects the identification accuracy and reliability, thus affecting the estimation accuracy of SOC. The hybrid pulse power characteristic (HPPC) test experimented is an off-line identification method. The model parameters are calculated by obtaining the variation law of the battery pack's closed-circuit voltage output response. The real-time performance is poor, so it cannot be applied for various working conditions [5]. Recursive least squares (RLS) method is a common identification algorithm based on online estimation to achieve high accuracy. For online identification of lithium-ion, data updating will lead to problems such as data saturation, and it is difficult to track the parameters of the time-varying system. Increasing the forgetting factor can increase the information content of new data and weaken the influence of old data, effectively avoid data protection phenomenon [6].

In this paper, a $30 \mathrm{Ah}$ rated lithium-ion phosphate battery was used as the experimental research object. Based on the mixed simplified electrochemical model, the model parameter variables were identified online by forgetting factor recursive least squares (FFRLS) method. Then Unscented Kalman Filter (UKF) is used to estimate SOC. Finally, the accuracy and feasibility of the method are verified by Federal Urban Driving Schedule (FUDS) experiment.

\section{Model establishment and parameter identification}

\subsection{Establishment of the mixed simplified electrochemical model}

The electrochemical model is a complex system, and some parameters are determined at the beginning of the design. Moreover, it will not change significantly into the entire battery aging cycle, so the accuracy of estimation is relatively high. However, due to the large number of parameters in the electrochemical model, it will take a lot of time to identify all parameters and the real-time performance is poor. Generally, the electrochemical model is simplified to be applied to SOC estimation of power cells. There are three commonly used simplified electrochemical models, namely Nernst

\footnotetext{
* Juqiang Feng: fjq5060912@126.com
} 
model, Shepherd model and Unnewehr model [6]. The above three models are based on experience and are suitable for some specific batteries. Combined with the above three models, a mixed simplified electrochemical model was constructed to improve the adaptability. The mixed simplified electrochemical model equation is shown in Eq. (1).

$$
\begin{aligned}
U_{o c}= & k_{0}-R * I-k_{1} * z+k_{2} / z \\
& +k_{3} \ln (z)-k_{4} \ln (1-z)
\end{aligned}
$$

$U_{o c}$ is the terminal voltage of the battery. $I$ is the battery charging and discharging current, which is negative when charging and positive when discharging. $R$ is the internal resistance of the battery. $z$ is SOC of the battery. $k_{0}, k_{1}, k_{2}, k_{3}$ and $k_{4}$ are the matching coefficients of the model.

\subsection{Model parameter identification}

The online parameter identification method uses the realtime data of current and voltage measured by the power battery to identify the parameters, so as to realize online updating of model parameters. There are many identification methods, such as RLS, genetic algorithm (GA), support vector machine (AVM) and so on. RLS method is developed based on adaptive theory and is one of the most commonly used methods for model parameter identification. However, it is difficult to obtain reliable estimation results with RLS for the system of continuous slow transformation. The least squares method based on forgetting factor can estimate the reliability of system parameters by inserting forgetting factor into the measured data.

Eq. (2) and (3) are obtained from the basic equation of FFRLS and Eq. (1).

$$
\begin{gathered}
y_{k}=\Phi_{k} \theta_{k}+e_{L S, k} \\
\left\{\begin{array}{l}
y_{k}=U_{o c} \\
\Phi_{k}=\left[\begin{array}{llllll}
1 & -I & -Z & 1 / Z & \ln (Z) & -\ln (1-Z)
\end{array}\right] \\
\theta_{k}=\left[\begin{array}{llllll}
k_{0} & R & k_{1} & k_{2} & k_{3} & k_{4}
\end{array}\right]^{T}
\end{array}\right.
\end{gathered}
$$

$\Phi_{k}$ represents the system data matrix. $\theta_{k}$ represents the system parameter matrix. $e_{L S, k}$ represents stationary zero mean white noise. To facilitate the distinction, the lower angle $k$ represents the corresponding data of $k$ sampling moments. The calculation flow of the algorithm is shown in Eq. (4).

$$
\left\{\begin{array}{l}
\mathrm{K}_{\mathrm{LS}, k}=\mathrm{K}_{\mathrm{LS}, k-1} \Phi_{k}^{T}\left[\Phi_{k} \mathrm{P}_{\mathrm{LS}, k} \Phi_{k}^{T}+\mu\right]^{-1} \\
\hat{\theta}_{k}=\hat{\theta}_{k-1}+\mathrm{K}_{\mathrm{LS}, k}\left[y_{k}-\Phi_{k} \hat{\theta}_{k-1}\right] \\
\mathrm{P}_{\mathrm{LS}, k}=\frac{1}{\mu}\left[\mathrm{I}-\mathrm{K}_{\mathrm{LS}, k} \Phi_{k}\right] \mathrm{P}_{\mathrm{LS}, k-1}
\end{array}\right.
$$

$\mu$ is the forgetting factor. When the value is 1 , Formula 4 degenerates into the traditional RLS. $\mathrm{P}_{\mathrm{LS}, k}$ is the error covariance matrix of the state estimate. $\mathrm{K}_{\mathrm{LS}, k}$ is the gain matrix of the algorithm.

This method constructs the real-time sampling of the current and voltage data of the power battery based on the input data. In this paper, the value of forgetting factor was set as 0.95 , the gain matrix and covariance matrix were calculated, and the parameters were identified and updated online, so as to realize SOC estimation and terminal voltage value prediction.

\subsection{Validation of the model}

In order to verify the validity of the online identification algorithm in this paper, FUDS operating condition experiment is designed. There are 22 working steps and 360 data points in one cycle. The experiment completed 19 cycles with a total of 7078 data points. Fig. 1 shows the comparison between the simulated and experimental values of the voltage at the charging and discharging terminals, and Fig. 2 shows the absolute error between the two.

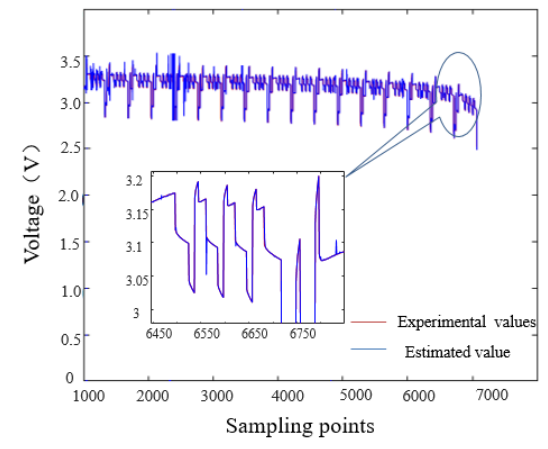

Fig.1. Comparison of experimental and simulation value

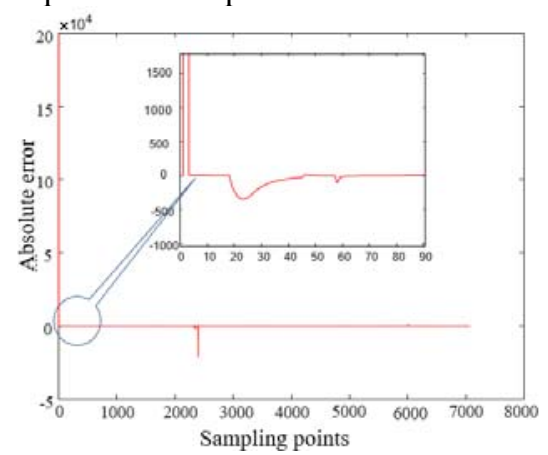

Fig.2. Absolute error between experimental and estimated

As can be known from the Fig.1, the experimental value of terminal voltage differs little from the simulation result. With the constant update of sampling point data, the simulation result of the system becomes more stable and robust.

As illustrated in Fig.2. Under the influence of the initial value, the error in the initial stage of simulation is large. But it soon converges and becomes stationary after 3 sampling points. At the same time, under the influence of the experimental environment, jitters were generated at 2350 and 6010 sampling points. Partial enlargement is shown in Fig.3. 


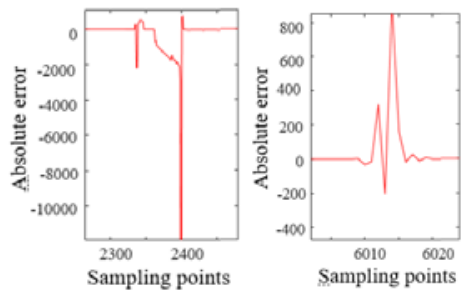

Fig.3. Local magnification of error results

In Fig.3, Due to the introduction of forgetting factor, the system tends to be stable after data updating with 50 and 10 sampling points respectively. Effectively avoiding the divergence and saturation of the system.

The curve diagram of parameter identification results is shown in Fig.4.

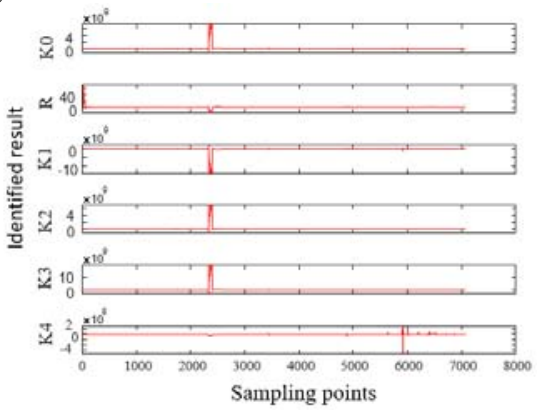

Fig.4. Parameter identification result

\section{Estimation of SOC}

\subsection{Unscented Kalman Filter (UKF)}

The internal electrochemical reaction of the cell is complex and has strong nonlinear property. The UKF abandoned the linearization of nonlinear functions and used Unscented Transform (UT) to deal with nonlinear transfer problems of mean and covariance, which can effectively overcome the problem of low estimation accuracy of the EKF [7].

Eq. (5) is a general expression of nonlinear discrete system.

$$
\left\{\begin{array}{l}
x_{k}=f\left(x_{k-1}, u_{k-1}\right)+\omega_{k-1} \\
y_{k}=h\left(x_{k}, u_{k}\right)+v_{k}
\end{array}\right.
$$

The specific process of UKF algorithm to estimate SOC of lithium battery is as follows:

1) Initialization:

$$
\hat{x}_{0}=E\left(x_{0}\right), P_{0}=\operatorname{var}\left(x_{0}\right)
$$

2) State prediction: $2 n+1$ sigma points are obtained by UT transformation of the optimal value of the system state variable at the time of $K-1$. The sigma points are substituted into the equation of state to obtain one-step prediction of the state variable. The way to construct the sigma points is shown in Eq. (7), and the time update of the state variable is performed according to the mean weight, as shown in Eq. (8).

$$
\begin{aligned}
& x^{i}=\hat{x}_{k-1}, i=0 \\
& \left\{x^{i}=\hat{x}_{k-1}+\left(\sqrt{(n+\lambda) P_{k-1}}\right)_{i}, i=1, \ldots n\right. \\
& x_{k-1}^{i}=\hat{X}_{k-1}-\left(\sqrt{(n+\lambda) P_{k-1}}\right)_{i-n}, i=n+1, \ldots 2 n \\
& \left\{\begin{array}{l}
X_{k \mid k-1}^{i}=f\left(X_{k-1}^{i}, u_{k-1}\right) \\
\hat{X}_{k \mid k-1}=\sum_{i=0}^{2 n} \omega_{m}{ }^{i} X_{k \mid k-1}^{i}
\end{array}\right.
\end{aligned}
$$

In Eq. (7) and (8), $i$ represents the ith column of the covariance matrix of the state variable. The mean weight $\omega_{\mathrm{m}}$ and variance weight $\omega_{\mathrm{c}}$ are calculated in formula 9 . After repeated debugging and verification, the parameters are set $k=2, \alpha=0.01, \beta=2$.

$$
\left\{\begin{array}{l}
\omega_{m}{ }^{0}=\frac{\lambda}{n+\lambda} \\
\omega_{\mathrm{c}}^{0}=\frac{\lambda}{n+\lambda}+1-\alpha^{2}+\beta \\
\omega_{m}^{i}=\omega_{c}^{i}=\frac{1}{2 *(n+\lambda)}, \quad i=1 \ldots 2 n
\end{array}\right.
$$

3) Update the covariance of the state variable:

$$
P_{x, k \mid k-1}=\sum_{i=0}^{2 n} \omega_{c}^{i}\left[x_{k \mid k-1}^{i}-\hat{x}_{k \mid k-1}\right]\left[x_{k \mid k-1}^{i}-\hat{x}_{k \mid k-1}\right]^{T}+Q_{k}
$$

4) Update of observed variables:

$$
\left\{\begin{array}{l}
y_{k \mid k-1}^{i}=h\left(x_{k \mid k-1}^{i}, u_{k}\right) \\
\hat{y}_{k \mid k-1}=\sum_{i=0}^{2 n} \omega_{m}{ }^{i} \mathrm{y}^{i}{ }_{k \mid k-1}
\end{array}\right.
$$

5) Update the error covariance:

$$
\left\{\begin{array}{l}
P_{y y, k}=\sum_{i=0}^{2 n} \omega_{c}^{i}\left[y_{k \mid k-1}^{i}-\hat{y}_{k \mid k-1}\right]\left[y_{k \mid k-1}^{i}-\hat{y}_{k \mid k-1}\right]^{T}+R_{k} \\
P_{x y, k}=\sum_{i=0}^{2 n} \omega_{c}^{i}\left[x_{k \mid k-1}^{i}-\hat{x}_{k \mid k-1}\right]\left[y_{k \mid k-1}^{i}-\hat{y}_{k \mid k-1}\right]^{T}
\end{array}\right.
$$

6) Kalman gain update:

$$
K_{k}=P_{x y, k} / P_{y y, k}
$$

7) State update and optimal covariance matrix:

$$
\left\{\begin{array}{l}
\hat{x}_{k \mid k}=\hat{X}_{k \mid k-1}+K_{k}\left(y_{k}-\hat{y}_{k \mid k-1}\right) \\
P_{x, k \mid k}=P_{x, k \mid k-1}-K_{k} P_{y y, k} K_{k}^{T}
\end{array}\right.
$$

\subsection{Results and discussion}

In order to verify the validity of the online identification algorithm in this paper, FUDS operating condition experiment is designed. The experimental current is shown in Fig.5. 


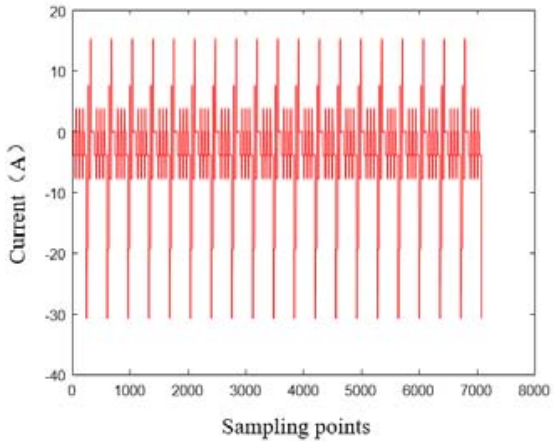

Fig.5. FUDS test current

After the test, the experimental data was imported into the simulation model. Online characterization parameters proposed in this paper and off-line characterization parameters of HPPC test were applied for SOC estimation based on UKF algorithm. The effects of SOC estimation were shown in Fig. 6.

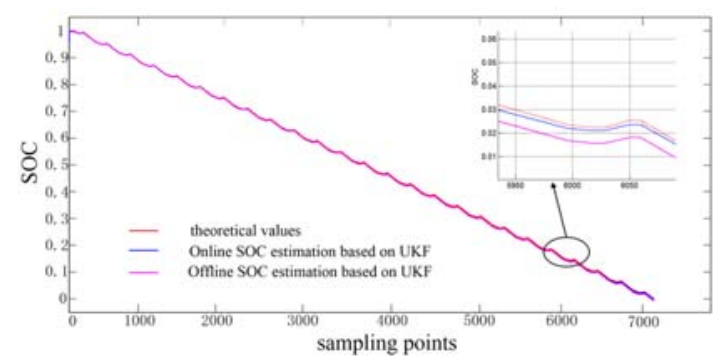

Fig.6. Comparison diagram of SOC estimation

As shown in Fig.6, the battery is charged and discharged and shelved in the FUDS working condition. Therefore, the SOC in the obtained SOC waveform decreases and increases. However, the following effect of the two algorithms is good. After local amplification, it can be seen that the online identification method proposed in this paper can converge rapidly and effectively, and approach the theoretical value steadily all the time.

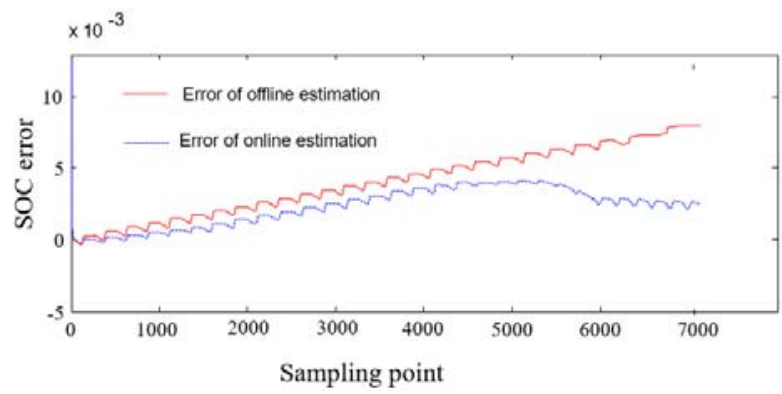

Fig.7. Comparison diagram SOC estimation error

Fig.7 shows the errors obtained by the two SOC estimation methods. Due to the influence of UKF algorithm, both methods have some accumulation errors. In this algorithm, battery model parameters change with the change of operating conditions are considered, and the model parameters are identified and updated online in real time. Therefore, reliable equation of state can be provided for SOC estimation. With the constant update of data, the SOC error obtained by the algorithm in this paper changes by leaps and bounds, and the final orientation is stable, with the error of about $0.9 \%$.

\section{Conclusion}

Firstly, the importance of accurate SOC estimation and the influence of model parameter identification accuracy on SOC estimation are described. Based on the mixed simplified model, FFRLS algorithm is used to realize the online identification and experimental analysis of model parameters. Simulation model was built based on HPPC off-line method and FFRLS online identification method, and UKF algorithm was used to realize SOC estimation of the two methods. According to the analysis results, the simulation model established in this paper is more accurate, and the stability error under FUDS condition is $0.9 \%$.

\section{Acknowledgements}

The authors would like to thank the Power supply and Control Engineering Technology Research Centre and Science Key Fund of Education Department of Anhui province (KJ2019A0692) for support.

\section{References}

1. Y. Diao, K. xie, S. Xiong, and X. Hong. Shuttle phenomenon-theirreversible oxidation mechanism of sulfur active material in Li-S batter. Journal of Power Sources, 2013,235(15):181-186.

2. Karsten Propp, Daniel J. Auger, Abbas Fotouhi, Monica Marinescu, Vaclav Knap, Stefano Longov. Improved state of charge estimation for lithiumsulfur batteries. Journal of Energy Storage, 2019, 25(12):1-13.

3. Yinjiao Xing, Wei He, Michael Pecht, Kwok Leung Tsui. State of charge estimation of lithium-ion batteries using the open-circuit voltage at various ambient temperatures. Applied Energy, 2014, 113(1):106-115.

4. Xiaosong $\mathrm{Hu}$; Shengbo Li; Huei Peng. A comparative study of equivalent circuit models for Li-ion batteries. Journal of Power Sources, 2012, 198(1): 359-367.

5. Petr Křivík. Methods of SoC determination of lead acid battery. Journal of Energy Storage, 2018, 15(2):181-186.

6. Shen P, Ouyang M, Lu L, Li J, Feng X. The coestimation of state of charge, state of health, and state of function for lithium-ion batteries in electric vehices. IEEE Transveh Technol, 2018,67:92-103.

7. SONG Xuanyu, HUANG Jingyao, DUAN Junqiang. Estimation of state of charge of battery based on adaptive recursive least squaress and unscented Kalman filtering. Electric Power Science and Engineering, 2019, 35(12):41-47. 